\section{Regards sur l'économie allemande}

Bulletin économique du CIRAC

$81 \mid 2007$

Varia

\title{
Compétitivité des territoires : état des lieux et perspectives
}

Isabelle Bourgeois

\section{OpenEdition}

\section{Journals}

Édition électronique

URL : http://journals.openedition.org/rea/649

DOI : $10.4000 /$ rea.649

ISBN : 978-2-8218-0858-4

ISSN : 1965-0787

\section{Éditeur}

CIRAC

Édition imprimée

Date de publication : 1 mai 2007

Pagination : 5-14

ISSN : 1156-8992

\section{Référence électronique}

Isabelle Bourgeois, «Compétitivité des territoires : état des lieux et perspectives », Regards sur l'économie allemande [En ligne], 81 | mai 2007, document 1, mis en ligne le 01 mai 2009, consulté le 30 avril 2019. URL : http://journals.openedition.org/rea/649 ; DOI : 10.4000/rea.649 


\section{Compétitivité des territoires : état des lieux et perspectives}

\section{Isabelle Bourgeois}

La croissance du PIB allemand a été de 2,9\% en 2006. Mais il ne s'agit là que d'une moyenne calculée en fonction des apports respectifs des Länder. Largement autonomes en matière de politique économique et budgétaire, ces 16 Etats membres qui composent la République fédérale se différencient par leur poids économique, le profil de leur économie, leur degré de compétitivité, le niveau de leur dette et, bien sûr, le taux de croissance.

Près de deux décennies après l'unification, le clivage est-ouest s'est fortement estompé. Globalement, les Länder est-allemands pèsent certes peu dans l'économie allemande, mais cela est dû essentiellement à leur petite taille en comparaison des trois poids lourds de l'ouest : Bavière, Bade-Wurtemberg et Rhénanie du Nord-Westphalie, qui contribuent pour une large moitié (56\%) au PIB allemand. Si on y ajoute la Hesse, numéro quatre, cette part s'élève aux deux tiers (65\%). Aujourd'hui, l'Allemagne se caractérise par un clivage nord-sud plus flagrant que jamais ; il devrait s'accentuer encore dans les années à venir du fait de la position centrale du pays dans l'espace économique européen. Si les régions du sud de l'Allemagne font partie depuis toujours de l'axe de compétitivité ouesteuropéen qui s'étend de Francfort/Main à Milan en passant par Munich, l'intégration des Etats est-européens dans l'UE bénéficie à des Länder comme la Saxe et la Thuringe au sud du territoire de l'ex-RDA, proches de la République tchèque et de la Hongrie voisine qui constituent un second axe de compétitivité européen nord-sud en plein développement, à l'est du premier. Les autres régions et Länder d'Allemagne, situés à l'écart de ces grands axes et ne disposant pas (ou moins) de compétences stratégiques pour l'avenir de leur économie, enregistrent des performances moyennes, voire faibles.

Les disparités s'accentuent ainsi entre les dynamiques territoriales, reléguant dans le peloton de queue de vastes ensembles régionaux par-delà les frontières des Länder, qu'ils soient situés à l'est (dans le Mecklembourg et la Saxe-Anhalt, par exemple) ou à l'ouest (Basse-Saxe, Hesse, Rhénanie-Palatinat). L'Allemagne s'est diversifiée au point que l'institut Prognos, qui vient de consacrer une étude à la compétitivité des districts régionaux (non des Länder), évoque même aujourd'hui une structure territoriale « à quatre vitesses ».

Une partie des disparités économiques entre Länder s'explique par la 'construction' de la RFA après 1945. La structuration des entités territoriales et politiques que sont les Länder s'est effectuée progressivement, sur la base d'identités provinciales préexistantes et fortement marquées comme dans le cas de la Bavière ou de Hambourg, mais bien plus encore au gré de la politique des Alliés dans leurs zones d'occupation respectives. C'est ainsi qu'a été créé le minuscule Land de Brême (une ville-Etat : Stadtstaat) : il s'agissait d'une enclave portuaire américaine dans la zone d'occupation britannique. Quant au Land de Bade-Wurtemberg, il a été créé en 1952, après la constitution de la RFA (1949), par voie de referendum, et réunit depuis des régions qui se situaient dans les anciennes zones d'occupation française et américaine. Les Länder est-allemands pour leur part ne sont nés qu'après la chute du Mur, par réanimation des identités provinciales qu'avaient gommées les régimes centralisateurs du $\mathrm{II}^{\mathrm{e}}$ Reich (ce qui valait

Les frontières actuelles des Länder sont le fruit de I'histoire 
également pour l'ouest) puis de la RDA. La Saxe et la Thuringe, au statut d'Etat libre (Freistaat) comme la Bavière, en sont les exemples patents. En ce qui concerne Berlin, la partie est, capitale de l'ex-RDA, a été réintégrée dans la ville-Etat de Berlin, aujourd'hui également capitale de la RFA réunifiée.

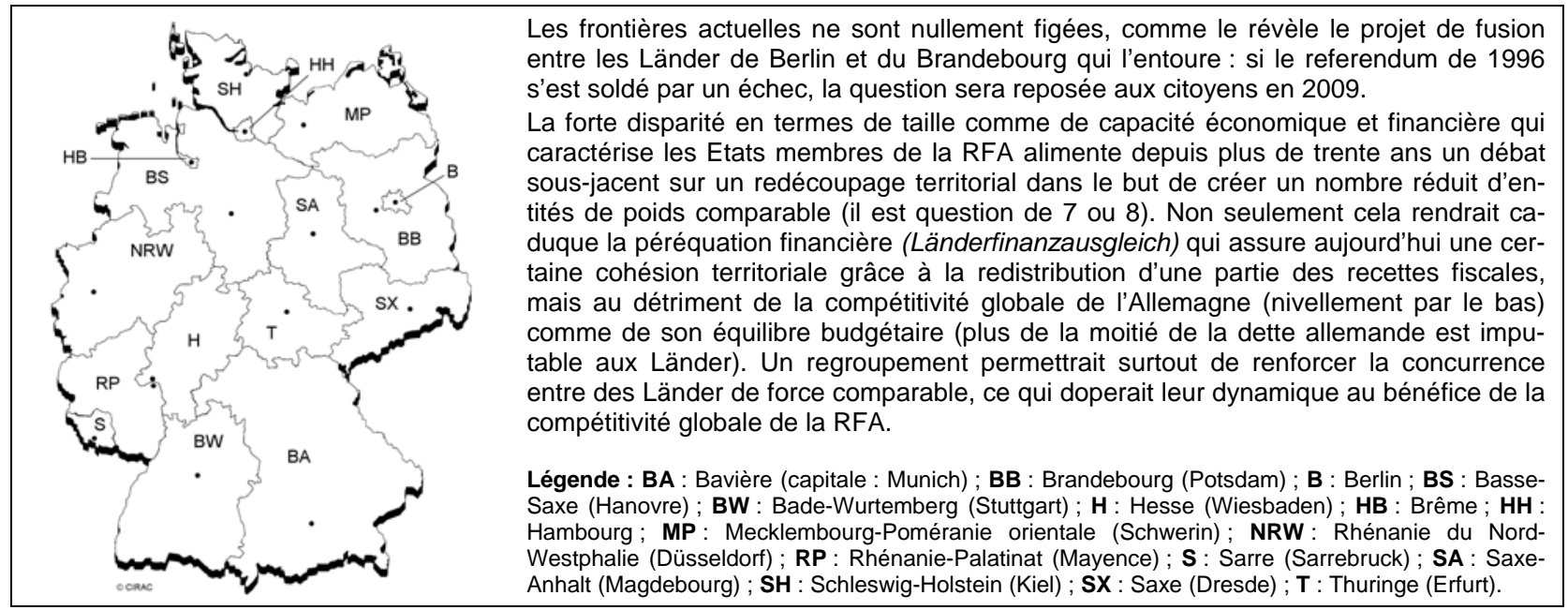

Les Länder industriels ont les meilleurs taux de croissance
En termes de croissance, le champion est la Saxe, un Land industriel de taille moyenne : elle a enregistré un taux de $+4,0 \%$ en 2006. L'année précédente, alors que la moyenne allemande s'établissait à $+0,9 \%$, la médaille revenait à la petite Sarre, industrielle elle aussi $(+2,8 \%)$ et elle aussi en restructuration. La riche Hesse, site tertiaire (financier) par excellence et quatrième économie d'Allemagne, présente pour sa part des performances moyennes : $+2,1 \%$ après un maigre $+0,4 \%$ en 2005. C'est que ce Land qui abrite la BCE se trouve pénalisé par le fait que le tertiaire y représente $75 \%$ des activités. Car les services ne s'exportent guère - à l'inverse des biens industriels, ce qui explique pourquoi le Bade-Wurtemberg se classe numéro deux en termes de croissance $(+3,5 \%$ en 2006). II est non seulement le site industriel allemand par excellence, mais aussi le leader européen de la construction mécanique et donc le leader allemand (la part de la construction mécanique allemande dans l'UE est de $39 \%$ ).

Les 16 Länder de la République fédérale

\begin{tabular}{|c|c|c|c|c|c|c|c|}
\hline & $\begin{array}{l}\text { Superficie }^{\text {a) }} \\
\text { en } \mathrm{km}^{2}\end{array}$ & $\begin{array}{l}\text { Habitants }^{\mathrm{a})} \\
\text { en millions }\end{array}$ & $\begin{array}{l}\text { Densitéa) } \\
\text { hab./ } / \mathrm{km}^{2}\end{array}$ & $\begin{array}{l}\mathrm{PIB}^{\mathrm{b})} \text { en } \\
\text { milliards } €\end{array}$ & Croissance $^{\text {b) }}$ & $\begin{array}{c}\text { PIB par } \\
\text { hab. }^{\text {b) }} \text { en } €\end{array}$ & $\begin{array}{c}\text { Part dans } \\
\text { RFA }^{\text {b) }}\end{array}$ \\
\hline Bade-Wurtemberg & 35752 & 10,7 & 300 & 337,2 & $+3,5 \%$ & 31400 & $14,6 \%$ \\
\hline Basse-Saxe & 47624 & 8,0 & 168 & 196,5 & $+2,7 \%$ & 24600 & $8,5 \%$ \\
\hline Bavière & 70552 & 12,5 & 177 & 408,3 & $+2,5 \%$ & 32700 & $17,7 \%$ \\
\hline Berlin & 892 & 3,4 & 3807 & 80,3 & $+1,9 \%$ & 23600 & $3,5 \%$ \\
\hline Brandebourg & 29479 & 2,6 & 87 & 49,5 & $+2,1 \%$ & 19400 & $2,1 \%$ \\
\hline Brême & 404 & 0,7 & 1641 & 25,3 & $+2,8 \%$ & 38100 & $1,1 \%$ \\
\hline Hambourg & 755 & 1,7 & 2309 & 86,0 & $+3,7 \%$ & 49200 & $3,7 \%$ \\
\hline Hesse & 21115 & 6,1 & 289 & 204,1 & $+1,8 \%$ & 33600 & $8,9 \%$ \\
\hline Mecklembourg-Poméranie & 23180 & 1,7 & 74 & 32,3 & $+2,1 \%$ & 19000 & $1,4 \%$ \\
\hline Rhénanie du Nord-W. & 34085 & 18,1 & 530 & 500,7 & $+2,8 \%$ & 27800 & $21,7 \%$ \\
\hline Rhénanie-Palatinat & 19853 & 4,1 & 204 & 100,3 & $+2,6 \%$ & 24700 & $4,4 \%$ \\
\hline Sarre & 2569 & 1,0 & 409 & 28,0 & $+2,0 \%$ & 26700 & $1,2 \%$ \\
\hline Saxe & 18415 & 4,3 & 232 & 88,7 & $+4,1 \%$ & 20800 & $3,8 \%$ \\
\hline Saxe-Anhalt & 20446 & 2,5 & 121 & 50,0 & $+3,8 \%$ & 20400 & $2,2 \%$ \\
\hline Schleswig-Holstein & 15799 & 2,8 & 179 & 69,7 & $+1,7 \%$ & 24600 & $3,0 \%$ \\
\hline Thuringe & 16172 & 2,3 & 144 & 45,8 & $+2,8 \%$ & 19700 & $2,0 \%$ \\
\hline RFA & 357093 & 82,4 & 231 & 2302,7 & $+2,8 \%$ & 28000 & $100,0 \%$ \\
\hline
\end{tabular}

Sources des données: Superficie et habitants: Statistische Ämter des Bundes und der Länder; PIB, croissance et part du PIB allemand (aux prix courants) : Arbeitskreis Volkswirtschaftliche Gesamtrechnungen der Länder. a) état au 31-12-2005 ; b) part du PIB, 2006 (état ; janvier 2007). NB : chiffres arrondis.

Les branches phares se concentrent dans quatre Länder
Les Länder ne se différencient pas seulement par leur taille, mais bien plus encore par la structure de leur économie, déterminante pour leur contribution au PIB 
allemand. Dans une économie allemande ouverte à l'international, et dont le principal moteur est l'exportation de biens et services industriels, les trois poids lourds industriels que sont la Rhénanie du Nord-Westphalie (au PIB comparable à celui des Pays-Bas, voir REA 62/03), la Bavière (voir REA 40/99) et le Bade-Wurtemberg (voir REA 74/05) jouent un rôle prépondérant. Ils sont le fief des branchesphares de l'industrie allemande: construction mécanique, automobile, électrotechnique et chimie. Ils se voient dorénavant défiés par la Saxe.

\section{Concentration régionale des secteurs-phares de l'industrie allemande}

Cette structuration territoriale de l'économie allemande est en grande partie le fruit de l'histoire : la Rhénanie du Nord-Westphalie et le Bade-Wurtemberg à l'ouest, la Saxe et la Thuringe à l'est, sont les berceaux de l'industrialisation allemande; de par leur profil, ces régions sont toujours spécialisées dans l'automobile, la construction mécanique ou la mécanique de précision et se caractérisent par un dense tissu de PME (même en Rhénanie du Nord-Westphalie).

Le site de Wolfsburg (Basse-Saxe), pour sa part, connu au XIX ${ }^{e}$ siècle pour la manufacture Büssing (camions), avait été choisi quasi ex nihilo comme site de production de la future "voiture du peuple " (Volkswagen) par une commission instituée par Hitler en 1937. La Saxe, du temps de la RDA, était au cœur du dispositif de production et de R\&D en microélectronique du régime; les anciens combinats Robotron et Mikroelektronik n'ont pas survécu, mais après l'unité allemande, le tissu d'activités s'est reconstitué sur des bases plus modernes. II en fut de même de l'optique de précision à léna en Thuringe (Zeiss), redynamisée après l'Unité. Quant à la région de Munich, elle doit son rang de métropole de l'électrotechnique à la construction du Mur de Berlin : Siemens, qui y avait son siège, avait alors préféré le transférer en des lieux plus sûrs.

Mais l'histoire a jeté aussi les bases de profils aujourd'hui obsolètes, comme le montrent les restructurations en cours dans la chimie en SaxeAnhalt (Bitterfeld, Leuna), ou dans ce qui reste d'industrie charbonnière et sidérurgique lourde dans le bassin de la Ruhr.

Légende : $\boxminus$ automobile ; $\widehat{O}$ chimie ; construction mécanique ; 艺 électrotechnique; $\hat{0}$ électronique et informatique.

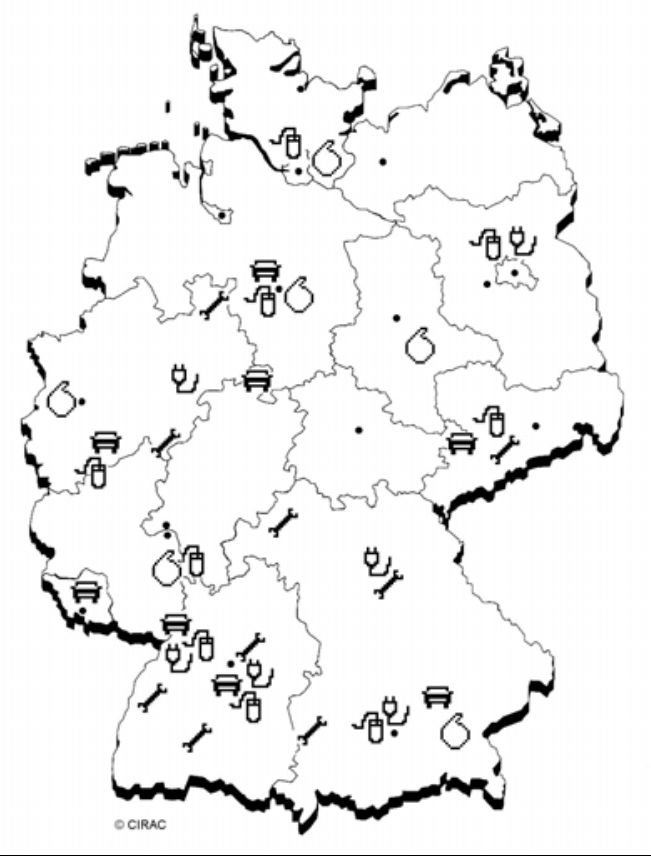

Structure de l'économie des Länder (2005) - part respective des secteurs (en \%)

\begin{tabular}{|c|c|c|c|c|c|c|c|}
\hline & $\begin{array}{l}\text { Agri- et } \\
\text { sylvi- } \\
\text { culture }\end{array}$ & Industrie & BTP & $\begin{array}{l}\text { Commerce, } \\
\text { gastronomie, } \\
\text { transports }\end{array}$ & $\begin{array}{l}\text { Crédit, bail, } \\
\text { services aux } \\
\text { entreprises }\end{array}$ & $\begin{array}{l}\text { Prestataires } \\
\text { publics et } \\
\text { privés }\end{array}$ & $\begin{array}{l}\text { Total PIB } \\
\text { (milliards } € \text { ) }\end{array}$ \\
\hline Bade-Wurtemberg & $0,80 \%$ & $34,26 \%$ & $4,16 \%$ & $15,66 \%$ & $26,29 \%$ & $18,83 \%$ & 294,11 \\
\hline Basse-Saxe & $1,85 \%$ & $26,26 \%$ & $4,25 \%$ & $26,26 \%$ & $24,72 \%$ & $23,72 \%$ & 172,61 \\
\hline Bavière & $1,11 \%$ & $26,71 \%$ & $3,93 \%$ & $16,08 \%$ & $31,55 \%$ & $20,61 \%$ & 359,60 \\
\hline Berlin & $0,14 \%$ & $15,24 \%$ & $3,16 \%$ & $16,47 \%$ & $33,69 \%$ & $31,30 \%$ & 71,17 \\
\hline Brandebourg & $2,14 \%$ & $19,69 \%$ & $5,21 \%$ & $18,99 \%$ & $25,66 \%$ & $28,33 \%$ & 43,38 \\
\hline Brême & $0,27 \%$ & $24,60 \%$ & $2,84 \%$ & $25,50 \%$ & $26,63 \%$ & $19,15 \%$ & 22,19 \\
\hline Hambourg & $0,19 \%$ & $15,80 \%$ & $2,02 \%$ & $27,07 \%$ & $37,19 \%$ & $17,71 \%$ & 74,85 \\
\hline Hesse & $0,50 \%$ & $21,34 \%$ & $3,41 \%$ & $19,17 \%$ & $36,91 \%$ & $18,67 \%$ & 181,02 \\
\hline Mecklembourg-Pom. & $2,83 \%$ & $13,37 \%$ & $5,56 \%$ & $21,06 \%$ & $24,81 \%$ & $32,40 \%$ & 28,58 \\
\hline Rhénanie du N.-W. & $0,63 \%$ & $25,61 \%$ & $3,40 \%$ & $18,34 \%$ & $29,28 \%$ & $22,72 \%$ & 439,62 \\
\hline Rhénanie-Palatinat & $1,60 \%$ & $28,04 \%$ & $3,76 \%$ & $17,40 \%$ & $25,21 \%$ & $25,21 \%$ & 88,25 \\
\hline Sarre & $0,24 \%$ & $30,45 \%$ & $4,12 \%$ & $14,84 \%$ & $27,66 \%$ & $22,68 \%$ & 24,73 \\
\hline Saxe & $1,08 \%$ & $22,42 \%$ & $5,66 \%$ & $16,57 \%$ & $27,25 \%$ & $27,03 \%$ & 76,84 \\
\hline Saxe-Anhalt & $1,70 \%$ & $22,57 \%$ & $5,70 \%$ & $21,21 \%$ & $20,66 \%$ & $28,18 \%$ & 43,51 \\
\hline Schleswig-Holstein & $1,66 \%$ & $18,64 \%$ & $3,41 \%$ & $21,68 \%$ & $29,26 \%$ & $25,32 \%$ & 61,85 \\
\hline Thuringe & $1,52 \%$ & $24,83 \%$ & $5,75 \%$ & $16,64 \%$ & $23,09 \%$ & $28,14 \%$ & 40,15 \\
\hline $\boldsymbol{R F A}$ & $0,98 \%$ & $25,45 \%$ & $3,89 \%$ & $18,08 \%$ & $29,24 \%$ & $22,36 \%$ & 2022,47 \\
\hline
\end{tabular}

Source des données : Arbeitskreis Volkswirtschaftliche Gesamtrechnungen der Länder (aux prix courants ; données publiées le 14-02-2007; état : août 2006). NB : calculs effectués par nos soins ; pourcentages arrondis.

Si les trois villes-Etats Berlin, Hambourg et Brême présentent une économie à dominante tertiaire, les services s'y distinguent par leur nature. Berlin héberge de nombreuses administrations dont les fédérations professionnelles et les lobbies qui se sont installés à proximité du gouvernement fédéral et du Bundestag. Les
Les villes-Etats se distinguent par la structure de leur tertiaire 
Mecklembourg : un Land rural et fief des centres d'appel

La Basse-Saxe présente la structure la plus complexe

sites portuaires Hambourg (premier port maritime d'Allemagne) et Brême (dont le port est situé dans la commune de Bremerhaven, une autre enclave à l'intérieur du Land de Basse-Saxe, mais rattachée administrativement au Land de Brême), se caractérisent par une prédominance du commerce et de la logistique. Si l'industrie n'y est pas absente, elle est directement liée aux activités portuaires, comme la construction navale ou l'agro-alimentaire ; ces Etats hanséatiques sont également les deux principaux sites de production d'Airbus outre-Rhin. Quant à Bremerhaven, il est le premier site allemand des éoliennes offshore.

D'autres Länder, étendus mais à faible densité de population, offrent un profil économique plus disparate, avec un secteur primaire plus développé qu'ailleurs. C'est le cas notamment du Mecklembourg, plat pays bordé au nord par la Mer Baltique, où $30 \%$ de la population vit dans des communes de moins de 2000 habitants et dont les deux tiers de la surface d'exploitation sont voués à l'agriculture. Mais cette ruralité, propice aux activités touristiques (l'île de Rügen est l'une des plus réputées d'Allemagne), n'exclut pas les activités industrielles comme l'agroalimentaire et surtout la construction navale (Rostock), ni encore moins les services de logistique : le Land est le fief allemand des centres d'appel et l'un des principaux centres de prestations postales sur le segment concurrentiel. Malgré son point fort qui est d'être une plateforme d'échanges avec l'espace baltique (via notamment la ville hanséatique de Greifswald), le Land n'en reste pas moins excentré, c'est-à-dire à l'écart des grands axes terrestres de communication transeuropéens, ce que reflète aussi son faible poids économique.

La Basse-Saxe limitrophe à l'ouest, second Land par la superficie après la Bavière, présente en Allemagne l'une des structures économiques les plus diversifiées, ce qui n'est pas étranger à sa topographie structurée par un réseau hydrographique sud-nord-ouest (des monts du Hartz et du Weserbergland vers la Mer du Nord) entrecoupé de canaux transversaux est-ouest dont le Mittellandkanal, tronçon principal d'un réseau de canaux est-ouest qui relie le Rhin à la Weser (estuaire : Brême), à l'Elbe (axe Hambourg-Prague) et à l'Oder. Cet axe de communication fluvial, aussi important que les liaisons routières et ferroviaires ParisVarsovie, passe comme celles-ci par Hanovre et Wolfsburg. Schématiquement, c'est dans la zone de ces infrastructures de communication que se situe l'essentiel de l'industrie, vouée principalement à l'automobile (VW à Wolfsburg et $\mathrm{Ha}$ novre), et aux TIC (Hanovre). C'est aussi à Hanovre que se trouve le premier site allemand de foires/expositions internationales (avant Francfort/Main). Au nord de cet axe se situent les plaines alluviales s'étendant jusqu'à la Mer du nord et la lande de Lunebourg, vouées à l'agriculture et à l'industrie agroalimentaire. La zone plus montagneuse au sud présente de fortes disparités: la vallée de la Leine (sud-nord) est à dominante agricole ; les marges occidentales de l'ancien Rideau de Fer sont à faible activité, sauf les monts du Hartz, région touristique. Deux pôles de hautes technologies et d'industrie s'y distinguent : la région de Brunswick à proximité du Mittellandkanal, classée la plus intensive en R\&D de I'UE (avionique et biotechnologies surtout) et qui abrite aussi l'une des plus anciennes universités techniques d'Allemagne (la Carolo Wilhelmina, créée en 1745). Et la ville de Salzgitter, le plus gros port fluvial de l'Allemagne du nord et $3^{\mathrm{e}}$ centre industriel de la Basse-Saxe où se concentrent industrie des métaux (sidérurgie, moteurs) et électronique automobile.

\section{Les Länder sont souverains quant à leur politique structurelle}

Les Länder sont des Etats souverains
Plus déterminante pour la diversité des profils des Länder est la politique économique que mènent leurs gouvernements. Car l'organisation fédérale de l'Allemagne leur confère de larges pouvoirs. La réforme du fédéralisme politique intervenue en août 2006 les accroît encore, mais sans modifier l'architecture de la répartition des pouvoirs entre les différents échelons territoriaux. Institutionnellement, les 16 Länder sont les collectivités territoriales constitutives de la RFA. Ils sont dotés chacun d'une Constitution propre, d'un parlement (Landtag) et d'un 
gouvernement : son chef, appelé ministre-président, est plus puissant qu'un ministre fédéral. Car ces Etats, souverains sur leur territoire, sont bien plus qu'une entité administrative 'déconcentrée' : ce sont de véritables Etats fédérés. Dans la marche des affaires de la Fédération (Bund), ils ont un pouvoir de co-décision via le Bundesrat, la chambre du Parlement allemand où siègent les représentants de leurs gouvernements et sans l'aval de laquelle aucune loi touchant aux prérogatives des Länder ne peut être adoptée (c'est le cas d'un grand nombre de textes). Ce pouvoir constitue la première facette de ce fédéralisme coopératif qui caractérise l'Allemagne, avec sa répartition à la fois fonctionnelle et sectorielle des pouvoirs entre les membres de l'ensemble solidaire qu'est la République fédérale.

La seconde facette en est la répartition sectorielle des compétences législatives. Ainsi, les Länder sont seuls souverains en matière d'éducation, de culture et de médias. Dans la plupart des autres domaines, il y a concurrence entre les compétences du Bund et celles des Länder: ces derniers sont habilités à légiférer " aussi longtemps et pour autant que la Fédération n'a pas fait par une loi usage de sa compétence législative » (art. 72, § 2 de la Loi fondamentale). Or dans les faits, et malgré un catalogue très restreint de compétences exclusives, le Bund se révèle le législateur dominant, la compétence concurrente jouant en sa faveur en vertu de la primauté du droit fédéral (art. 31 de la Loi fondamentale). Au fil du temps, cette compétence concurrente avait fini par mener à une centralisation rampante des pouvoirs qui, en rognant la latitude des Länder sur leur territoire, était l'une des principales sources d'immobilisme de l'Allemagne et de nivellement des performances des Länder. Cette dérive a été en partie corrigée par la réforme du fédéralisme politique qui a clarifié la répartition des pouvoirs législatifs entre Bund et Länder. Elle a en outre réduit la part des lois fédérales soumises à approbation du Bundesrat, passée de $60 \%$ à un peu moins de $40 \%$ désormais.

La troisième facette du fédéralisme concerne la répartition fonctionnelle des pouvoirs. "Les Länder exécutent les lois fédérales à titre de compétence propre », stipule l'art. 83 de la Loi fondamentale. Autrement dit: ce sont les Länder qui exercent seuls le pouvoir administratif. Le Bund n'a en effet pas d'administration centrale, sauf dans les rares domaines qui relèvent de sa compétence régalienne exclusive : défense et affaires étrangères pour l'essentiel aujourd'hui. Les Länder mettent donc en œuvre les lois soit pour le compte du Bund (administration déléguée), soit au titre de leurs compétences propres. Dans d'autres cas encore, Bund et Länder coopèrent pour l'exécution de tâches communes, dont la liste a été nettement réduite lors de la réforme du fédéralisme politique. Le fait que les Länder disposent de compétences législatives somme toute résiduelles au regard du catalogue établi par la Constitution ne leur interdit donc nullement de mener leur propre politique de développement structurel. Bien au contraire, puisque ce sont eux qui disposent du pouvoir administratif, ce qui les habilite à gérer leurs affaires en pleine autonomie, que ce soit pour le compte de la Fédération, au titre des tâches communes, ou dans l'intérêt de leur site économique.

De plus, le Bund et les Länder étant «autonomes et indépendants les uns des autres dans leur gestion budgétaire » (art. 109, § 1 de la Loi fondamentale), les Länder sont souverains pour gérer la part des recettes fiscales qui leur revient après la péréquation financière à laquelle sont soumis Bund, Länder et communes (Finanzausgleich). Quelque $70 \%$ des recettes fiscales perçues outre-Rhin sont ainsi partagées entre les divers échelons territoriaux. Certes, les Länder n'ont qu'une faible autonomie pour définir une fiscalité originale, et peu d'impôts leur reviennent en propre (comme l'impôt sur les successions). Mais ils sont pleinement souverains en ce qui concerne la gestion de leurs finances, n'étant soumis qu'au contrôle législatif de leur Landtag et réglementaire de leur Cour des comptes. Cette liberté leur confère la latitude requise pour mener la politique de développement régional définie par chacun.

Elle présente toutefois un inconvénient majeur dont on a pris pleinement la mesure voici une dizaine d'années seulement, au moment de la définition des règles
Leurs compétences législatives sont certes résiduelles, ...

... mais ce sont eux qui exercent le pouvoir administratif

Ils sont souverains en matière budgétaire

II n'existe pas de Pacte de stabilité interne à l'Allemagne 
Redistribution financière pour assurer l'homogénéité des conditions de vie

Une réforme pour plus de concurrence et une meilleure solidarité

Chaque Land mène sa propre politique de cohésion territoriale liées à l'adoption de la monnaie unique. En effet, les critères relatifs à la dette dans le Pacte de stabilité et de croissance ne sont contraignants que pour le Bund. Or celui-ci ne maîtrise que moins de la moitié des dépenses et engagements (les Länder réalisent quelque $40 \%$ des dépenses publiques allemandes). Au plan intérieur, il n'existe pas d'équivalent allemand au Traité de Maastricht (seule s'applique la législation adoptée par la plupart des Länder sur le modèle de l'art. 115 de la Loi fondamentale qui interdit un recours au déficit d'un montant supérieur à celui des investissements). Cette absence de contrainte pesant sur les dépenses a abouti au fait que presque tous les Länder sont lourdement endettés, sauf trois, au budget aujourd'hui non seulement équilibré, mais aussi sans nouvel endettement à brève échéance : la Bavière, la Saxe et, depuis 2006, le Mecklembourg. Plus de la moitié de la dette allemande reste imputable aux Länder, ce qui a forcé le lancement de la réforme du fédéralisme financier actuellement en débat. Et incité récemment quatre Länder à engager une politique de rigueur pour présenter un budget équilibré en 2011 : Bade-Wurtemberg, BasseSaxe, Brandebourg et Saxe-Anhalt. D'autres, comme Hambourg, ont commencé à réduire drastiquement leurs dépenses.

Ce laxisme budgétaire se nourrit par ailleurs d'une dérive qu'a connue le fédéralisme financier au fil des décennies. L'objectif du partage des ressources consiste en théorie à assurer l'homogénéité des conditions de vie (gleichwertige Lebensverhältnisse ; art. 72, § 2 de la Loi fondamentale) sur l'ensemble du territoire de la RFA, c'est-à-dire à garantir l'équité des chances entre les 16 Länder - et non pas l'égalité, ainsi qu'a dû le rappeler le Tribunal constitutionnel fédéral dans un arrêt rendu en 1999 et dans lequel il établit le principe constitutionnel de "l'interdiction du nivellement » (Verbot der Nivellierung). Le problème ne se situe pas tant au plan de la répartition verticale du montant global des ressources fiscales allemandes entre les échelons territoriaux, dans le cadre de laquelle la quote-part de chaque Land lui est attribuée au prorata de son nombre d'habitants. II se situe au niveau horizontal, dans le mécanisme de solidarité jouant entre des Länder de poids disparate et qui vise à atténuer les inégalités de richesse : le Länderfinanzausgleich. Dans ce cadre, une partie des recettes fiscales revenant aux Länder 'les plus riches' (donateurs) est redistribuée aux 'moins riches' (bénéficiaires) afin d'assurer à chacun une capacité financière s'élevant à au moins $95 \%$ de la moyenne. Le total ainsi redistribué s'élevait à près de 7 milliards $€$ en 2005.

Si ce mécanisme a bénéficié à une Bavière sous-développée au sortir de la guerre et si, conjugué au Pacte de Solidarité Aufbau Ost de même qu'aux fonds structurels européens, il profite à des économies dynamiques comme la Saxe ou la Thuringe, il s'est révélé contre-productif pour la compétitivité de Länder comme Berlin (voir REA 79/06), mais aussi Brême, de loin le plus endetté de tous avec une dette de près de $19000 €$ par tête, ou la Sarre (9 $600 €$ ). Plus généralement, il tasse la croissance en réduisant la compétition entre sites: les 5 donateurs (Bade-Wurtemberg, Bavière, Hambourg, Hesse et Rhénanie du Nord-Westphalie) se voyant privés d'une partie des fruits de leur politique ; les récipiendaires (tous les autres) n'étant pas réellement incités à l'efficience. Selon les experts, le Länderfinanzausgleich en l'état actuel coûterait près d'un point de croissance à l'Allemagne (voir REA 52-53/01). II s'agit dorénavant de trouver un nouvel équilibre afin de redynamiser la concurrence entre sites tout en préservant la nature solidaire du fédéralisme financier ; le principe lui-même n'est pas en cause.

L'objectif d'homogénéité, corollaire de la cohésion territoriale, s'applique pareillement aux régions à l'intérieur de chaque Land. Sur son territoire, chaque gouvernement mène donc la politique qu'il juge utile pour développer la croissance et la stabilité de son économie, la prospérité des citoyens, des entreprises et des régions. Cela inclut politique industrielle, énergétique, éducative, technologique, culturelle, aménagement du territoire et création d'infrastructures, programmes d'aides à la création d'entreprises ou de développement structurel régional. La Bavière, par exemple, consacre quelque $12 \%$ de son budget (36 milliards $€$ en 2007) au seul soutien aux PME, aux infrastructures et à l'innovation. Autonome dans ses do- 
maines de souveraineté, chaque Land l'est également dans la mise en œuvre de la politique fédérale ou des missions communes. Tout l'art consiste dès lors pour chaque gouvernement à y intégrer intelligemment ses propres orientations et priorités. C'est là un facteur primordial pour l'identité territoriale.

Le gouvernement d'un Land a donc la possibilité de mener sa propre politique économique - au sens allemand du terme. Son rôle, tout comme d'ailleurs celui du gouvernement fédéral, consiste en effet essentiellement à créer et entretenir un cadre favorable aux activités : à l'émergence d'initiatives, à l'investissement, à la création d'entreprises et à l'emploi, sans oublier le développement de la qualité de vie. Et un gouvernement ne peut définir ces orientations générales pour son Land qu'en s'appuyant sur d'autres acteurs autonomes: communes, chambres de commerce et d'industrie, chambre des métiers, universités, fédérations, entreprises, etc. Car, selon le principe de subsidiarité, ce sont eux les véritables acteurs économiques sans qui aucune politique n'est possible à l'échelon supérieur. L'effort politique principal d'un gouvernement en matière de développement de son site économique se fondera donc sur une approche de bottom up propice à l'émergence des initiatives des acteurs privés locaux et visera à en impulser la coordination à l'échelle du Land, tout en mettant en œuvre les axes de la politique fédérale qu'il a lui-même en partie contribué à définir. C'est cet ensemble d'implication à divers échelons, d'impulsions actives, de coordination des moyens et des initiatives qu'on désigne communément par le terme de Standortpolitik.

II revient donc à chaque Land de donner corps aux grands objectifs d'équilibre économique communs à l'ensemble de l'Allemagne comme aux siens propres. L'originalité des choix faits par chaque Land sera bien sûr fonction des orientations idéologiques de la majorité élue, de chaque gouvernement en place. La Standortpolitik est donc aussi le moyen pour chacun de ces Etats de cultiver sa singularité et de se distinguer dans cette course aux résultats que se livrent les Länder dans un pays où, fondamentalement, la concurrence est considérée comme une saine émulation, qu'il s'agisse d'entreprises ou de territoires. La concurrence entre les Länder se révèle par là même aussi un des facteurs-clefs de la cohésion nationale, puisqu'elle promeut la confrontation des intérêts, préalable aux négociations nécessaires à la prise de décisions conjointes.

Or dans l'approche allemande du système économique, pour que la concurrence puisse pleinement produire ses effets positifs sur la collectivité, elle doit être régulée. Cette orientation ordo-libérale est largement partagée par toutes les forces politiques. La diversité des choix effectués par les Länder concourt ainsi au processus permanent de réflexion et d'ajustement national. La cohésion collective est en outre assise sur le principe de l'auto-régulation: dans cette concurrence entre sites, les parties prenantes ont en effet obligation de respecter un comportement visant à préserver l'unité fédérale (bundesfreundliches Verhalten). Ce principe, qui a force constitutionnelle, répond au souci institutionnalisé de garantir l'intérêt général dans le respect de la singularité des intérêts régionaux.

La marge de manœuvre des Länder est donc très grande. Et c'est du dynamisme de chaque Land, entretenu par la compétition, que dépend la prospérité collective du site Allemagne. Qu'un des 'poids lourds' voie faiblir son économie, et c'est l'Allemagne qui se trouve sous-performante. A l'inverse, la bonne tenue des affaires dans un Land accroît la prospérité de tous. C'est pour cela aussi que les Länder se livrent à un benchmarking permanent afin de déceler leurs forces et faiblesses et d'y apporter les réponses politiques requises.

Les politiques de compétitivité menées par les Länder sont donc diverses, construites sur des structures et des cultures régionales distinctes, bien sûr, mais également déterminées par les choix des électeurs et l'orientation idéologique de chaque gouvernement. La continuité politique est dès lors un facteur décisif pour l'évolution de l'économie d'un Land. Trois cas sont particulièrement révélateurs à cet égard : la Bavière (CSU ; majorité absolue depuis 1962), le Bade-Wurtemberg (où le CDU est au gouvernement depuis 1952, en coalition tantôt avec le SPD ou
Standortpolitik : la coordination des initiatives et acteurs

La concurrence entre sites promeut la cohésion nationale...

... en conciliant intérêt général et intérêts particuliers

Une pratique constante du benchmarking

La continuité politique favorise la compétitivité territoriale 
le FDP) et la Rhénanie du Nord-Westphalie (fief du SPD, dans des coalitions diverses, notamment avec les Verts, jusqu'en 2005).

\section{Etat libre de Bavière : lente et systématique construction de la compétitivité}

Le ministre-président Edmund Stoiber se place, comme ses prédécesseurs, dans le prolongement de l'action d'un Franz Josef Strauss qui fut à la tête du gouvernement bavarois de 1978 à 1988. Ce dernier, en qualité de ministre fédéral des Finances sous la première grande coalition (1966-69), co-rédigea avec son homologue de l'Economie, Karl Schiller (SPD), la Loi de promotion de la stabilité et de la croissance (1967) qui guide toujours l'action de tout gouvernement allemand.

Cette continuité a permis de transformer en 40 ans un Land rural et déshérité, sans ressources énergétiques notables, en un site industriel high tech, ce que traduit la devise adoptée dans les années 1990: "l'alliance entre l'ordinateur portable et la culotte de peau ». Cette transformation, favorisée par une structure administrative centralisée (cas unique en Allemagne), s'est effectuée en cinq étapes successives, mais aux effets cumulatifs : l'accueil massif de réfugiés allemands issus d'Europe centrale (années 1950), une politique de localisations industrielles avec pour point de cristallisation l'installation à Munich du siège de Siemens qui a eu pour effet, par grappage, le développement de clusters industriels (années 1960), l'adoption d'une Loi sur le soutien aux PME (Mittelstandsförderungsgesetz) qui servira de modèle aux programmes de soutien adoptés par les autres Länder et le Bund (années 1970), une politique conséquente de développement de la R\&D et des nouvelles technologies, notamment les biotechnologies (années 1980), suivie d'une réorientation ciblée vers l'innovation high tech (années 1990).

A cela se sont ajoutées une politique systématique de désenclavement et une politique énergétique centrée sur le nucléaire (quelque $70 \%$ de l'approvisionnement du Land) et l'hydroélectrique (la Bavière fournit près de la moitié de l'approvisionnement allemand). L'effort intense d'investissement, possible grâce au Finanzausgleich, s'est accompagné d'un effort particulier consenti aux ressources humaines : le baccalauréat bavarois est le plus prisé d'Allemagne, les universités cumulent les labels « élite » et " excellence ", les salariés figurent parmi les mieux qualifiés et le taux de chômage est l'un des plus faibles (7,8\% en 2006).

Mais la centralisation s'est parfois accompagnée d'un interventionnisme peu conforme aux usages allemands (sauvetage d'entreprises ou secteurs obsolètes), menant aussi à un pilotage industriel pas toujours en adéquation avec la réalité du marché ni du potentiel d'innovation régional (lancement trop uniforme, pas assez ciblé, de pôles de compétitivité). Aujourd'hui, le principal risque pour le Land réside dans une trop grande confiance dans ses acquis.

\section{Bade-Wurtemberg : une culture industrieuse décuplée par la mise en réseau des compétences}

Ce Land, de création tardive, a dû construire son identité, contrairement à la Bavière millénaire. II l'a fait en misant sur la tradition de créativité et d'innovation de ses habitants (Karl Benz, Gottlieb Daimler, Robert Bosch pour ne citer que les plus célèbres de ses inventeurs historiques) comme sur ses atouts indéniables en termes de qualité de vie : les agglomérations et pôles d'activité sont certes denses, disséminés sur l'ensemble du territoire, mais sans nuire au caractère naturel de l'environnement. Aujourd'hui, l'effort de R\&D représente $4 \%$ du PIB du Land (voire $7 \%$ dans la région de Stuttgart). Si le Land héberge de grands groupes comme Carl Zeiss, il est le fief par excellence des PME, dont nombre de ces champions mondialement réputés comme Stihl ou Kärcher.

La politique structurelle de ce Land, dont la seule ressource naturelle notable est le capital humain, est discrète par nature et conforme à la réputation de 'fourmis industrieuses' de ses habitants. Elle suit deux axes. D'une part la promotion de cette culture créative grâce à un intense effort de formation professionnelle (ses habitants sont les mieux qualifiés d'Allemagne : un habitant sur dix est diplômé de l'enseignement supérieur ; le taux de chômage est le plus faible avec $7 \%$ seulement en 2006) et de transfert des technologies et savoirs essentiellement vers les PME (c'est dans ce Land que s'est créée la Fondation Steinbeis, acteur majeur mais pas unique de la diffusion de l'intelligence économique). D'autre part, l'aménagement d'un cadre favorable à ces pôles de compétitivité qui se sont constitués spontanément comme un écosystème au fil du temps autour des piliers de l'industrie locale : automobile, construction mécanique, biotechnologies et chimie.

La politique structurelle des gouvernements se place donc délibérément au service des acteurs économiques. II est vrai que le Land se présente aujourd'hui comme un pôle de compétitivité pratiquement d'un seul tenant. Dès lors, sa politique de compétitivité se résume à cette formule de son ministre de l'Economie : "l'innovation naît dans un processus où l'économie, la science, la formation et la société agissent en réseau, et dont le centre est l'individu en tant que porteur des savoirs ». Si le Bade-Wurtemberg est bien armé pour la société du savoir, il doit, tout comme la Bavière, miser plus désormais sur l'avenir.

\section{Rhénanie du Nord-Westphalie : reconversion grâce à une révolution technologique}

Si ce Land structuré par le Rhin et la Ruhr, très divers du fait des provinces qu'il rassemble (la Rhénanie est catholique, la Ruhr protestante et la Westphalie mixte) n'est plus le «pays noir » qu'il fut jusque dans les années 1970, et si son industrie est aujourd'hui tertiarisée à un haut degré, il le doit à une politique de reconversion originale : du charbon aux TIC via le show biz. Berceau historique de la cogestion au cœur du « modèle rhénan » et théâtre en 1959 du Congrès de Bad Godesberg où fut définie la socialdémocratie, sa tradition SPD a contribué à une transition en douceur, alors même que l'emploi industriel (et minier) chutait brutalement. En contrepartie, le Land (qui hébergeait la capitale allemande d'avant l'Unité : Bonn) a cherché à développer ses forces et son potentiel : la forte concentration de médias, la présence de l'opérateur historique des télécommunications, celle de la Deutsche Bahn se conjuguent à un réseau d'infrastructures de communication d'une densité rare, et ce dans un Land qui occupe une position centrale dans l'espace économique européen.

Trois temps se distinguent dans sa politique structurelle : jusqu'à la fin du $X X^{e}$ siècle, il s'agissait de compenser les déficits structurels par un soutien aux activités en déclin (notamment dans la Ruhr) et le développement de secteurs nouveaux (TIC); ensuite, il a fallu entretenir et soutenir le potentiel d'innovation existant : au soutien sectoriel ou territorial (la ville de Gelsenkirchen dans la Ruhr a un taux de chômage de quelque $17 \%$ ) a donc succédé le soutien ciblé des pôles à fort potentiel. Mais cette politique de mise en réseau et de grappage restait pilotée par les pouvoirs publics et, surtout souffrait d'un financement trop indifférencié, menant à une mauvaise allocation des ressources alors même que le Land est lourdement endetté. Le changement de gouvernement en 2005 (arrivée au pouvoir d'une coalition CDU/FDP) a enclenché un recentrage plus systématique sur les pôles d'excellence, misant résolument sur les synergies public-privé et acceptant désormais l'existence de fortes disparités territoriales.

La reconversion du Land n'est pas achevée. Reste aussi à remédier au point faible du site qui se traduit par un taux de chômage de $12 \%$ en 2006 : la faible performance globale de son système éducatif - et universitaire. Seule émerge la RTWH d'Aix-la-Chapelle, au cœur de l'Euregio Meuse/Rhin où se trouve également le centre de recherche de Jülich. 
L'originalité des politiques structurelles que permet l'indépendance d'action des Länder explique comment la Saxe, Land est-allemand, est devenue un de ces "paysages fleuris » qu'invoquait le chancelier Kohl lors de l'unification. Certes, elle ne 'pèse' que l'équivalent de Berlin ou Hambourg dans le PIB allemand, mais son potentiel est loin d'être épuisé. Surtout, cet Etat libre doit sa compétitivité au fait que, dès 1994, le ministre-président d'alors, Kurt Biedenkopf (CDU) rompt radicalement avec la politique globale et indifférenciée de soutien à la transformation qu'est l'Aufbau Ost (voir REA 71/05), en ayant décelé l'un des premiers les effets pernicieux sur la compétitivité. Elle ne prenait en effet pas assez en compte les spécificités régionales. Sa réorientation est en débat dans le cadre de la réforme du fédéralisme financier et de la fin prochaine des aides européennes.
La transformation de la Saxe est le fruit d'une politique originale

\section{La Saxe, challenger de la Bavière}

La Saxe dispose, tout comme le Bade-Wurtemberg, d'une longue culture industrieuse que le régime de la RDA avait essayé de maintenir, mais avec des résultats mitigés. Après 1990, c'est sur ces fondations et la présence d'un dense tissu de PME que le Land établit sa politique de transition/restructuration, menant de front modernisation des activités industrielles et stratégie d'innovation. C'est ainsi que le Land est (re)devenu un grand site automobile (des Trabant aux Passat), a (re)dynamisé sa construction mécanique et qu'il arbore fièrement le qualificatif de "Silicon Saxony »: Dresde est aujourd'hui le premier cluster européen voué à la microélectronique (la ville accueillait, du temps de la RDA, le combinat Robotron).

Ce (re)positionnement s'est effectué d'une part grâce à une politique budgétaire rigoureuse qui a porté ses fruits : depuis 2006 , le Land présente un budget équilibré. D'autre part, grâce à une priorité absolue accordée à l'investissement productif pour créer les conditions permettant de générer et dynamiser l'activité.

Cette politique est à l'opposé de celle pratiquée par les autres nouveaux Länder qui, le plus souvent, utilisent les fonds de l'Aufbau Ost et les aides européennes pour combler leurs trous budgétaires - une pratique abusive qui alimente les débats, posant la question du bien fondé d'une politique de cohésion qui repose trop exclusivement sur une logique de redistribution indifférenciée.

La forte internationalisation de l'économie saxonne (près de la moitié des exportations des nouveaux Länder lui est imputable) qui tire sa croissance est le résultat de cette politique originale, inscrite elle aussi dans la continuité. Le fait que le Land soit aujourd'hui gouverné par une grande coalition CDU/SPD n'en a pas modifié l'approche. Sur ce fond, l'élargissement de l'UE laisse aussi présager un partage des tâches affiné entre une production orientale plutôt low-tech pour l'instant et le high-tech saxon. Reste à réduire le chômage (le taux dépasse encore $18 \%$ en 2006) et, surtout, à enrayer le vieillissement démographique accentué par l'exode des jeunes grâce notamment à une politique de qualifications plus en accord avec le potentiel de compétitivité du Land.

Si les Länder présentent une grande diversité, au sein même de chacun existent de fortes disparités. Le Brandebourg qui entoure Berlin, par exemple, ne comprend qu'une région dynamique : la ceinture du Land capitale, avec une concentration d'activités high-tech dans l'agglomération de Potsdam. La région de Francfort-sur-Oder à la frontière polonaise souffre des conséquences d'une politique de localisations industrielles trop volontariste et généreusement subventionnée qui avait cherché à recréer, sur les ruines du combinat sidérurgique d'Eisenhüttenstadt qui produisait la moitié de l'acier de la RDA, des activités sectorielles identiques (Usinor, Motorola) - en négligeant le fait que ces pôles y avaient été érigés ex nihilo par le régime de RDA, c'est-à-dire sans pouvoir s'intégrer dans un tissu d'activités préexistant. Quant aux activités dans le reste de ce Land faiblement peuplé, elles se partagent entre agriculture et tourisme, surtout dans les marges proches des régions à faible activité du sud du Mecklembourg et du nord-est de la Saxe-Anhalt.

Si ces disparités sont en partie liées à la restructuration des économies est-allemandes après l'Unité et si elles y sont de ce fait plus prononcées qu'à l'ouest, elles sont loin d'être la spécificité des nouveaux Länder. Le nord-est rural de la Hesse, par exemple, qui avait été coupé de la Thuringe par le Rideau de Fer, fait partie de ces régions accusant un retard économique, au même titre que le nord du Schleswig-Holstein à la frontière danoise ou le nord-est de la Bavière à la frontière tchèque. Ces régions n'ont jamais franchi le pas vers des activités dynamiques, d'autant qu'elles sont loin des grands axes de communication allemands et intraeuropéens. La question des infrastructures est en effet cruciale pour le développement économique local ou régional, comme le révèle la tendance à l'intégration de pôles d'activités distants entre eux, mais situés à proximité des grands axes. L'exemple-type en est celui des régions de Stuttgart et du Rhin-Main qui se densifient, préfigurant une tendance à la conurbation et à la concentration des activités comparable à celle de la Ruhr. C'est le cas aussi des agglomérations de
Au sein des Länder, les disparités régionales sont fortes... 
Dresde, Potsdam et léna, en plein essor grâce à leur situation sur les axes BerlinHanovre et Dresde-léna-Erfurt La structure établie des activités joue elle aussi un rôle fondamental dans ces évolutions : ce sont surtout les régions où domine une industrie low-tech qui présentent aujourd'hui une faible compétitivité. Ces régions en déclin se concentrent au nord du Main, et à l'ouest. C'est ainsi que s'esquisse une Allemagne à " 4 vitesses ": l'ensemble sud et sud-ouest est de loin le plus compétitif ; les performances du nord sont moyennes ; à l'est, quelques pôles au fort dynamisme se profilent dans un ensemble plutôt morose.

\begin{tabular}{|c|c|c|}
\hline Dynamique comparée ... & ... et forces actuelles... & ...des régions allemandes \\
\hline & & $\begin{array}{l}\text { Ces cartes comparent les forces et dynamiques des } 439 \text { dis- } \\
\text { tricts administratifs allemands pour lesquels l'institut Prognos } \\
\text { a collecté ses données auprès notamment des offices statis- } \\
\text { tiques du Bund et des Länder, de l'Agence fédérale pour } \\
\text { l'emploi, de l'Office des brevets, de la GfK ou de l'institut } \\
\text { ZEW. Le palmarès est établi sur la base d'une série d'indi- } \\
\text { cateurs comprenant notamment l'évolution démographique, } \\
\text { celle du marché de l'emploi, la croissance, le taux de créa- } \\
\text { tion d'entreprises, l'intensité de l'effort de R\&D, le nombre de } \\
\text { brevets déposés et la présence d'autoroutes. } \\
\text { Légendes : } \\
\text { 1. Dynamique : les régions ayant fait preuve du plus fort dynamisme au } \\
\text { cours des derrieres années figurent en noir sur cette carte, les moins } \\
\text { dynamiques en blanc. } \\
\text { 2. Forces : les régions les plus performantes figurent en noir sur cette } \\
\text { carte, les moins performantes en blanc. } \\
\text { Source des cartes : Prognos AG, Zukunftsatlas } 2007 \text { (www.prognos.com). }\end{array}$ \\
\hline
\end{tabular}

Dans une ALlemagne RedeVEnUe Competitive, LA VITALITE des LÄNDER revêt une importance décisive pour asseoir la croissance sur des bases durables. Une partie de leurs politiques de compétitivité se concentre sur les clusters qui bénéficient maintenant d'aides plus ciblées. Mais l'innovation technologique ne suffit pas. Le cadre des activités doit devenir encore plus incitatif : la plupart des Länder a engagé une modernisation de l'administration dans le sens d'une plus grande efficience et d'une proximité accrue avec les entreprises. Dans l'économie du savoir, la qualification des ressources humaines est un enjeu stratégique, et les Etats allemands ont commencé à réformer leurs systèmes de formation. Mais leur latitude d'action dans un environnement global en mutation se heurte aujourd'hui aux limites de leurs compétences législatives et budgétaires. Car la centralisation rampante des pouvoirs aux mains du Bund les prive de la souplesse requise pour adapter chacun le cadre réglementaire commun aux besoins spécifiques de sa propre économie. De ce fait, elle rend la politique du Land peu lisible aux yeux de ses citoyens, ce qui contribue à nourrir leur méfiance envers le monde politique. Quant à la solidarité financière qui lie les Länder, elle inhibe leur croissance en bridant la dynamique propre à chacun. Seule une réforme en profondeur des règles du jeu du fédéralisme politique et financier permettra de redynamiser la concurrence entre des sites exposés à la mobilité des facteurs de production au sein de la RFA, mais sans la possibilité d'y apporter la réponse adéquate. Cette réforme, en cours de discussion, implique l'acceptation des disparités territoriales, corollaire d'un retour au dogme originel au fondement du fédéralisme allemand comme de son ordre économique et social : le principe d'équité des chances.

\section{Indications bibliographiques :}

BerTelsmanN-Stiftung, Die Bundesländer im Standortwettbewerb 2005, Gütersloh, 2006 Cernavin O. et al., Cluster und Wettbewerbsfähigkeit von Regionen, Berlin, 2005

Fuest C., KeRvian D. et Welter P., Wirtschaftliche Freiheit in den deutschen Bundesländern, Friedrich-Naumann-Stiftung, novembre 2007 (www.fnst.org)

IW ConsuLt, Viertes Bundesländerranking, Cologne, septembre 2006 (www.iwconsult.de)

MC KINSEY, Projektbericht Perspektive-Deutschland 2005/06, Düsseldorf, 2006

ProGnos AG, Zukunftsatlas 2007, étude publiée en coopération avec le quotidien Handelsblatt. 